\title{
Investigations for broadband internet within high-speed trains
}

\author{
I. Gaspard and G. Zimmermann \\ T-Systems, BU Technologiezentrum, Am Kavalleriesand 3, 64295 Darmstadt, Germany
}

\begin{abstract}
Broadband IP-based multimedia services are rapidly growing and there is the trend to use these services everywhere. Due to the fact that passengers in long distance high speed trains have a very comfortable environment to use their own terminal equipment, e.g. laptops with IEEE $02.11 \times$ WLAN capability, it seems to be very promising to deliver broadband internet into trains. The challenge is the land-train connection where a high bit data rate link, e.g. $1 \mathrm{Mbps}$ in average in up- and downlink, has to be provided to enable several users within the train broadband internet access at the same time even at high speeds of the train. This paper describes a systematic approach starting at basic propagation measurements in a typical train environment and ending up with lab investigations by means of hardware channel simulation for different existing radio technologies which are candidates for the land-train connection.
\end{abstract}

\section{Introduction}

Market studies show that WLAN in trains has an even bigger potential than airport hotspots (BWCS report, 2003) for the coming years. A first key finding is that the market for trainbased wireless internet services is six times bigger than that for airport hotspots. The second main finding is that laptop users spend more than 12.6 billion $h$ on trains per year but just under two billion hours at airports. Thus it seems very attractive to provide broadband internet to the passengers of long distance high speed trains. In principle the solution is as shown in Fig. 1.

The passenger uses his or her own terminal with WLAN capability and is thus connected within a WLAN inside the train. The access points of the train WLAN infrastructure are connected to an universal train communication gateway which provides a wireless broadband link to one or more landside access networks. The challenging requirement for the train-land connection is twofold: first it has to be broadband, e.g. 1 Mbps each up- and downlink, to be able to carry the traffic generated of several passengers at the same time. Second the performance of this link has to be of reasonable performance even at very high speeds, e.g. $300 \mathrm{~km} / \mathrm{h}$.

Correspondence to: I. Gaspard

(ingo.gaspard@t-systems.com)

\section{Channel sounder measurements}

A versatile representation of the real characteristics of the mobile radio channel is given by the measured broadband channel impulse responses. These channel sounder measurements measured in typical reception scenarios can be used as a basis for radio system link performance investigations as well as for coverage planning investigations as given in Fig. 2.

The time-consuming and costly field measurements have to be done only once. Variation of parameters, like investigations at different velocities of the mobile terminal, can be considered by post-processing of the measured impulse responses.

\subsection{Field measurement setup}

To provide a realistic basis for further investigations channel sounder measurements were done with a RUSK WLL channel sounder (see: http://www.medav.de). Measurements were taken for two different transmitter positions A and B, see Fig. 3. In both experiments the mobile receiver was moved along a track in parallel to the railway track. For transmitter position A metal structures like poles and electrical power lines of the train infrastructure may have an impact onto wave propagation whereas for position B always a line of sight condition was given.

The main parameters of the channel sounder measurement experiments are given in Table 1. The distance between successive impulse responses was chosen to fulfil the spatial sampling theorem and thus allowing Doppler spectra derivation.

Figure 4 shows two photographs of the field measurement environment. The metallic train infrastructure towering into the line of sight for transmitter position A can clearly be seen.

\subsection{Evaluation of field measurements}

To verify the assumption that the metal infrastructure of the train track has an impact onto radio wave propagation the median values of path loss out of sections of $1 \mathrm{~m}$ length corresponding to $\approx 40$ impulse responses along the measurement track were analyzed for transmitter position A and B. Figure 5 shows the distribution of path loss for the two cases 


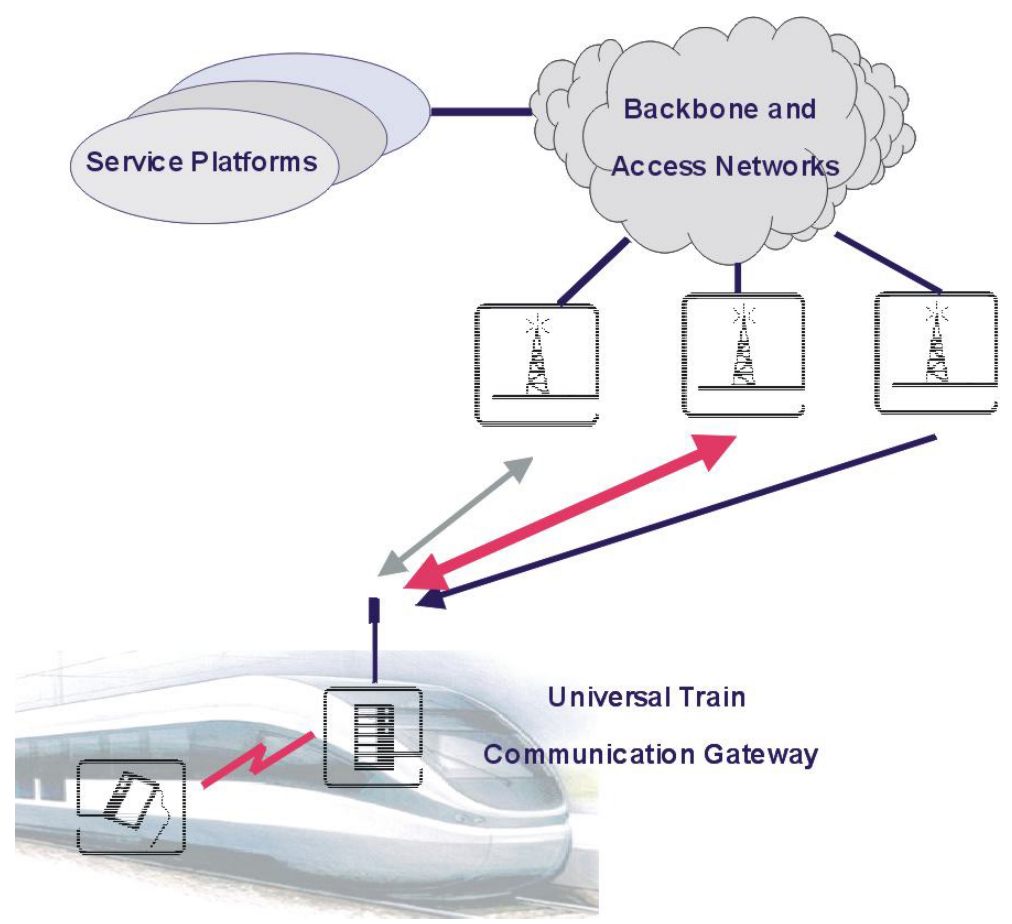

Fig. 1. Broadband internet in train - principle solution.

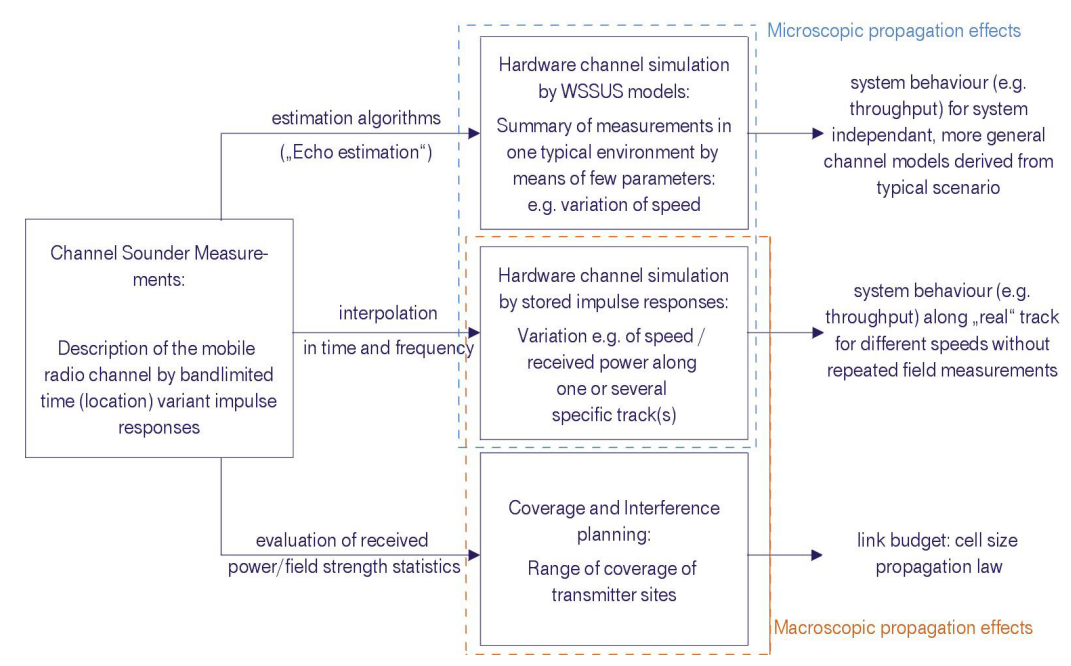

Fig. 2. Channel sounder measurements as the basis of further investigations.

Table 1. Main parameters of channel sounder experiments.

\begin{tabular}{ll}
\hline Carrier frequencies & $1.8 \mathrm{GHz} / 5.2 \mathrm{GHz}$ \\
\hline Measurement bandwidth & $22 \mathrm{MHz}$ \\
\hline Max. length / resolution of impulse response & $25.6 \mu \mathrm{s} / 25 \mathrm{~ns}$ \\
\hline Distance between successive impulse responses & $2.46 \mathrm{~cm}$ \\
\hline TX antenna height /gain & $9.7 \mathrm{~m} / 7.5 \mathrm{dBi}$ \\
\hline RX antenna height /gain & $1.7 \mathrm{~m} / 2.15 \mathrm{dBi}(1.8 \mathrm{GHz}), 0 \mathrm{dBi}(5.2 \mathrm{GHz})$ \\
\hline Distances between RX and TX & $40 \mathrm{~m} \ldots 400 \mathrm{~m}$ \\
\hline
\end{tabular}




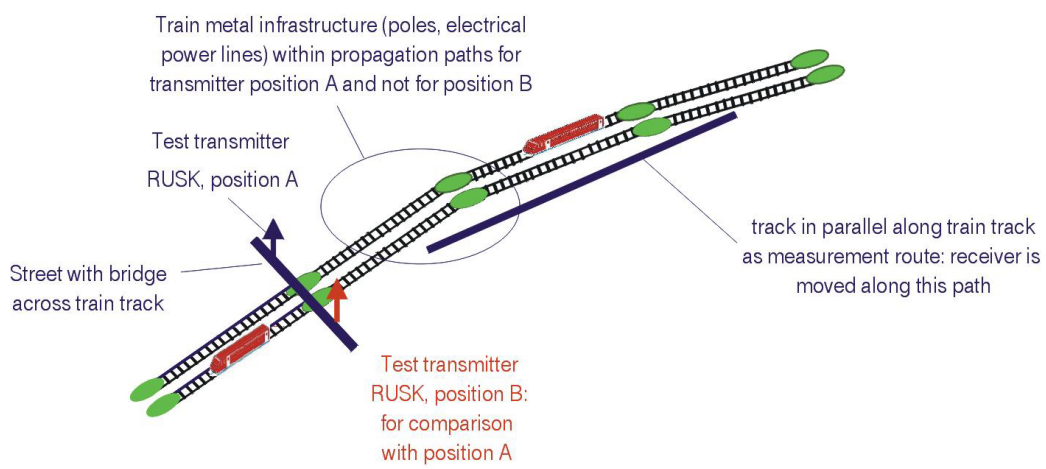

Fig. 3. Field measurement setup with different transmitter positions A and B.

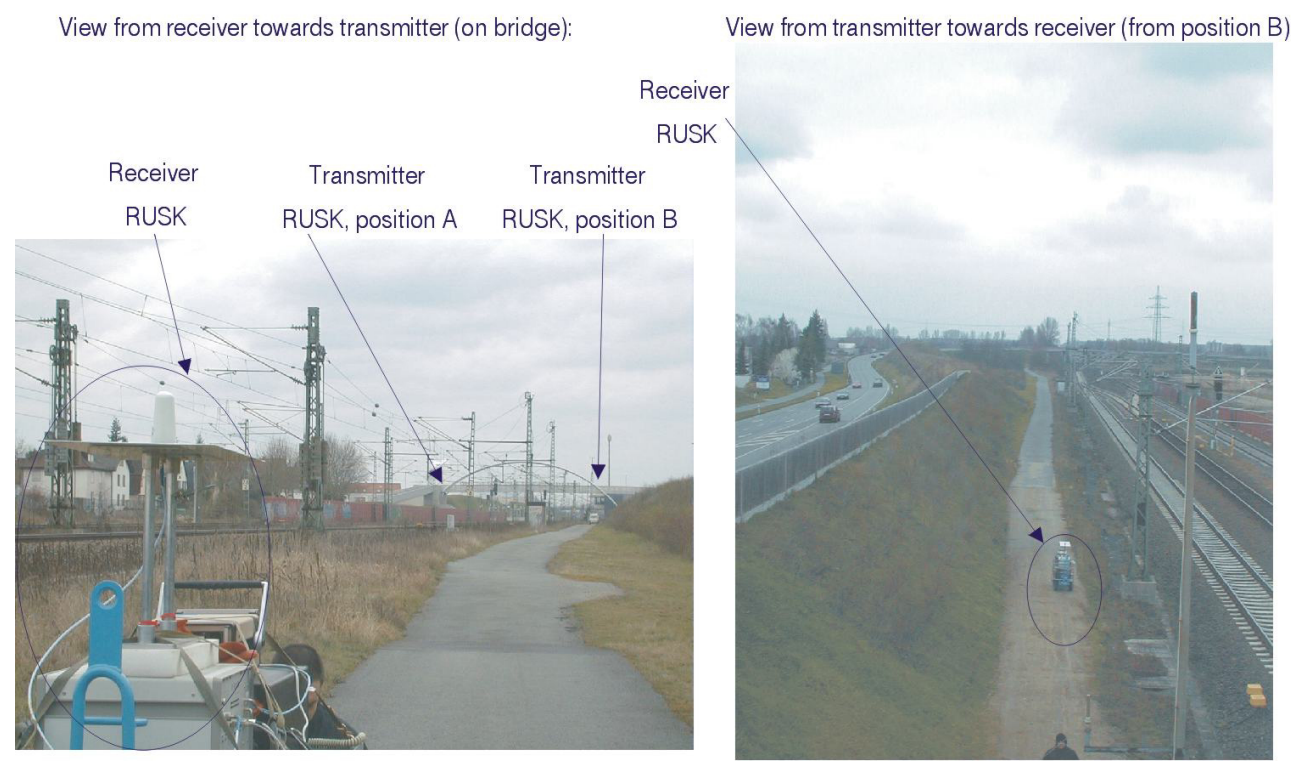

Fig. 4. Field measurement environment: view from RX towards TX antennas positions (left), view from TX antenna position B towards receiver (right).

with and without metal structures reaching into the line of sight between RX and TX. It can clearly be conducted that the presence of the metal structures for transmitter position B causes an increased path loss.

For further analysis WSSUS models were derived for successive blocks consisting of 64 impulse responses each by the methods developed in U. Martin (1994). The evaluation of estimated far field paths given in Table 2 shows that by the presence of metal structures in the line of sight between TX and RX more paths are estimated due to the more complicated propagation by additional diffraction and scattering.

Out of all WSSUS models estimated along the measurement run for $1.8 \mathrm{GHz}$ carrier frequency and TX position A three new simple two-path WSSUS models were derived as typical representative models and are given in Table 3 . These models are only differing in the power ratio of the first to second path and are thus differing in frequency selectivity.

\section{Measurement based lab investigations}

Several different existing radio technologies were investigated by means of hardware simulation of the radio channel. The main important performance measures were the throughput as a function of maximum Doppler and thus speed and the sensitivity which determines at the end the density of base stations along the track to have coverage.

In Fig. 6 a generic lab setup including the channel simulator is depicted.

In the following some measurement examples are given.

Figure 7 shows the throughput performance for UMTS FDD in downlink in case of TCP/IP traffic over $384 \mathrm{kbps}$ bearer for different simulated radio channels at constant speeds as a function of receiver input power. The "constant" channel corresponds to the AWGN channel whereas the two "2 path models" are derived from the measurements, see Table 3. The channel for the "Rice/Ratio $1 \mathrm{~dB}$ " is a frequency flat Rice channel with a power ratio of direct to 


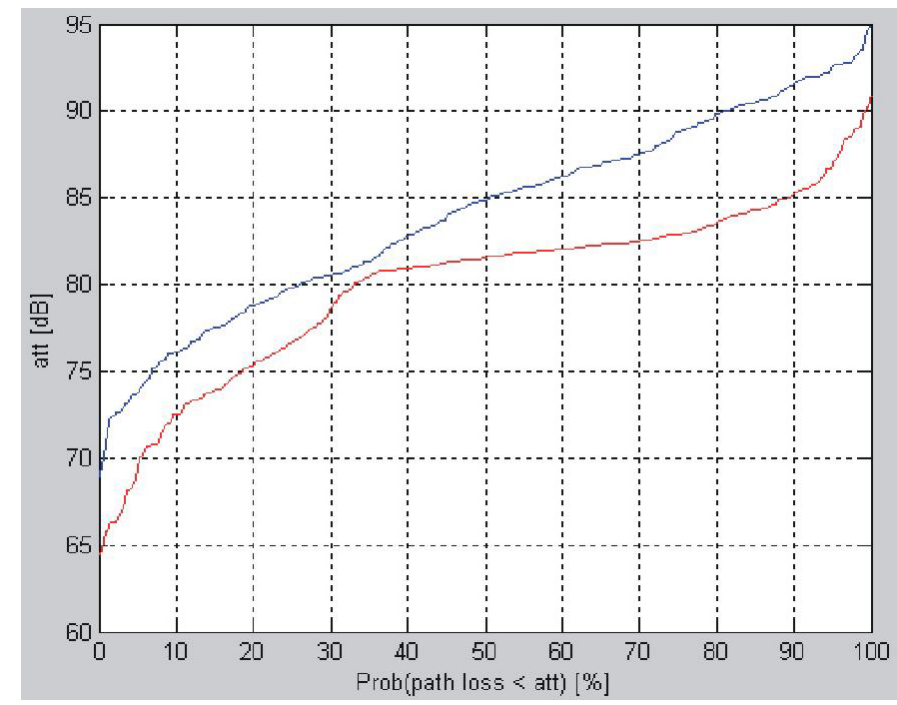

Fig. 5. Distribution of path loss (or attenuation) for TX position A/with metal structures (blue) and B/without metal structures (red).

Table 2. Evaluation of estimated WSSUS models.

\begin{tabular}{lllll}
\hline Number of paths in WSSUS models (@ $1.8 \mathrm{GHz})$ & 1 & 2 & 3 & $>=4$ \\
\hline Distance RX-TX:216 m . . 400, without metal structures (\%) & 17.7 & 82.3 & 0 & 0 \\
\hline Distance RX-TX:216 m ... 400, with metal structures (\%) & 12.4 & 38.9 & 41.2 & 7.5 \\
\hline
\end{tabular}

Table 3. Two-path WSSUS models derived from measurement campaign @ $1.8 \mathrm{GHz}$.

\begin{tabular}{lccc}
\hline Model \# & 1 & 2 & 3 \\
\hline Rice factor of first path & & $10 \mathrm{~dB}$ & \\
\hline Rice factor of second path & $5 \mathrm{~dB}$ & $10 \mathrm{~dB}$ & $15 \mathrm{~dB}$ \\
\hline Power ratio first to second path & \multicolumn{3}{c}{$0.2 \mu \mathrm{s}$} \\
\hline Delay between first and second path & & \\
\hline Doppler spectra for first and second path & Classical Jakes, in addition: Dirac at $+\mathrm{f}_{\text {Dmax }}$ \\
\hline
\end{tabular}

scattered components of $1 \mathrm{~dB}$. The "Rural Area" channel is a 6 path COST207 channel which is the most frequency selective channel in comparison to the aforementioned channels. As can be seen from the curves throughput is nearly constant with decreasing input power down to $-105 \mathrm{dBm}$. For the frequency flat channel "Rice/Ratio $1 \mathrm{~dB}$ " as well as for the frequency selective channel "Rural Area" only $64 \mathrm{kbps}$ are possible at the given speeds whereas for the two channels derived from measurements more than $300 \mathrm{kbps}$ are reached.

A similar measurement result of an IEEE802.11b link where data rate against input power was measured for different radio channels is given in Fig. 8. Throughput drops down already at moderate receiver input power values, e.g. for the "Rice/Ratio $1 \mathrm{~dB}$ " channel an input power of at least $-85 \mathrm{dBm}$ is necessary to allow at throughput of $1 \mathrm{Mbps}$. This input power value is a very sensitive to the applied channel. Thus and because of EIRP limitation in the $2.4 \mathrm{GHz}$ band a very high density of access points along the track would be necessary for full coverage.
As a further example throughput measurement results for a preliminary IEEE802.16 (WiMAX) system are given in Fig. 9. In this figure the TCP/IP throughput as function of speed in downlink is given for the system operated at $3.5 \mathrm{GHz}$ carrier frequency occupying a bandwidth of 3.5 $\mathrm{MHz}$ for different modulation schemes of the OFDM subcarriers as well as for different radio channels at a constant receiver input power of $-65 \mathrm{dBm}$. For most of the channels and most of the modulation schemes a fast decreasing throughput with increasing speed can be observed. Only for the most robust modes and for the frequency flat channels there is less dependence on speed. Especially channel estimation seems to be a critical item due to the fact that IEEE802.16 focused on fixed and not on mobile wireless access. It has to be noticed that IEEE802.16e enhancements will allow also enhanced channel estimation as well as it will include handover mechanisms. 


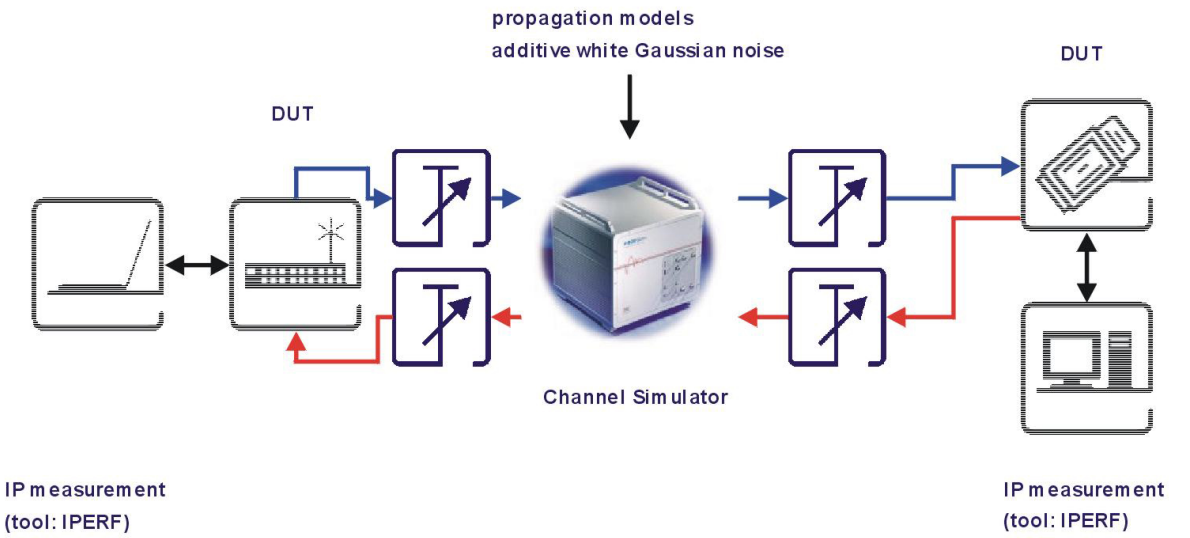

Fig. 6. Generic lab setup for channel simulator measurements. DUT= Device Under Test.

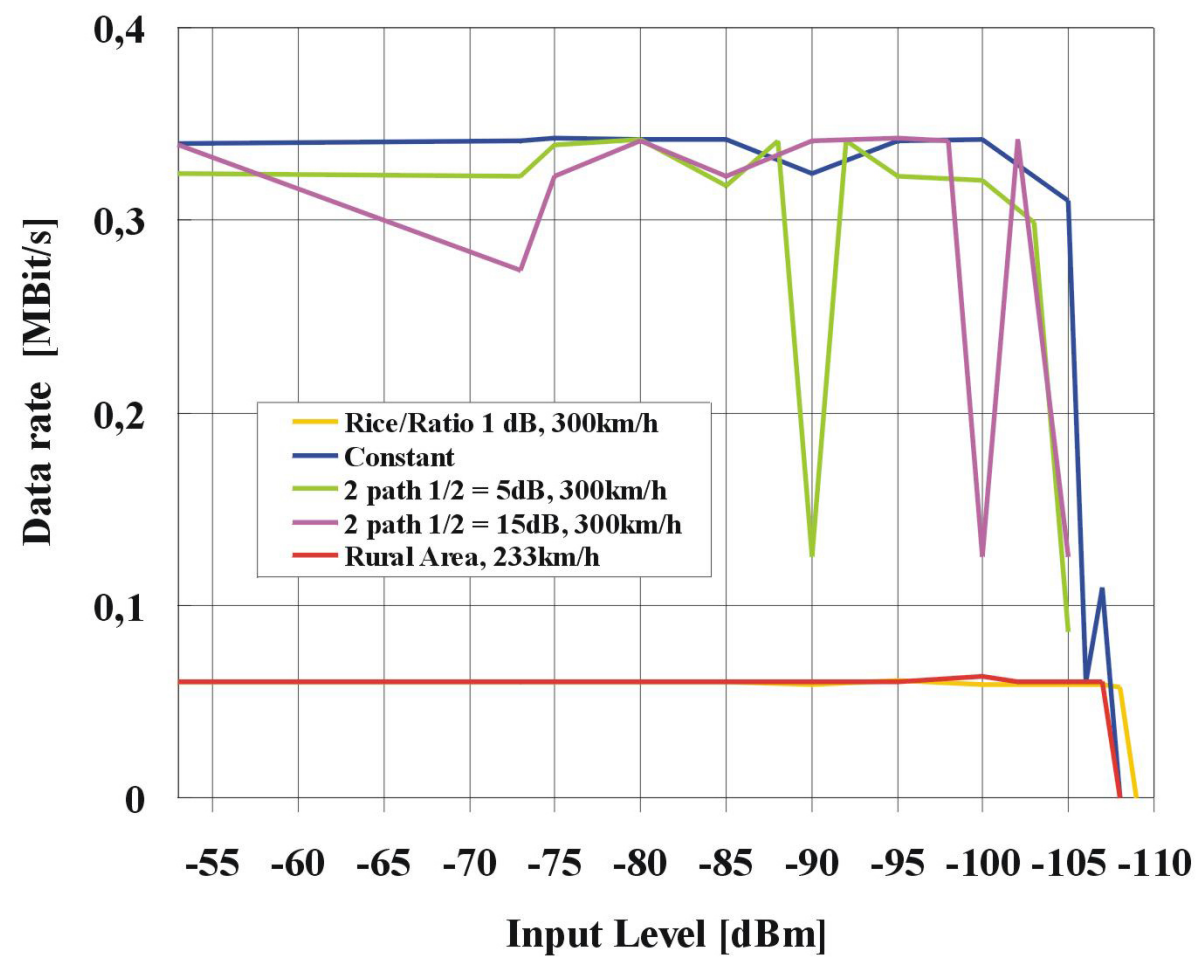

Fig. 7. Measured TCP/IP throughput in UMTS FDD downlink as a function of receiver input power for different channels.

\section{Conclusion}

In this paper we showed a systematic approach to investigate existing radio systems in a high speed train application which was not their primary application area. Starting with realistic channel impulse response measurements which allow a general description of the radio channel properties WSSUS models were derived. It could be shown measurement evaluation that typical infrastructure like poles and electrical power lines along the tracks have an impact onto the propagation characteristics of the electromagnetic waves between transmitter and receiver. By means of hardware simulation measurements were performed regarding IP throughput on the basis of the derived WSSUS models. For comparison reasons the performance for 'Rural Area' and frequency flat channel models were also investigated. It turns out that the UMTS system is performing well for various channels but delivers only relatively low data rates. The IEEE802.11b system is able to deliver high data rates in principle but these are fast decreasing for realistic multipath channels at high velocities. This holds even for high $\mathrm{S} / \mathrm{N}$ values. Also the preliminary IEEE802.16 system which was investigated is not well suited for high speed applications. Enhancements are expected for the near future because mobility aspects like enhanced channel estimation capabilities and handover mechanisms are currently in the IEEE standardization process. 


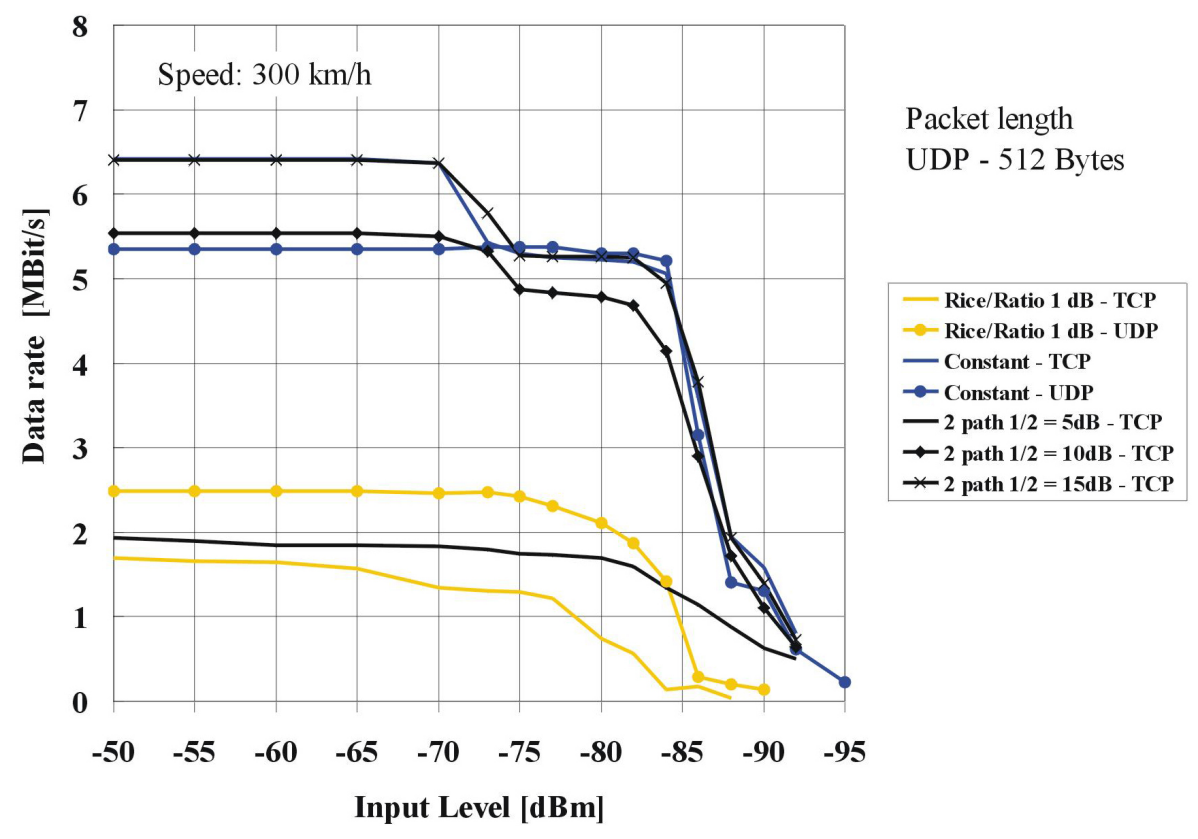

Fig. 8. Measured TCP/IP and UDP throughput in IEEE802.11b as a function of receiver power for different channels.

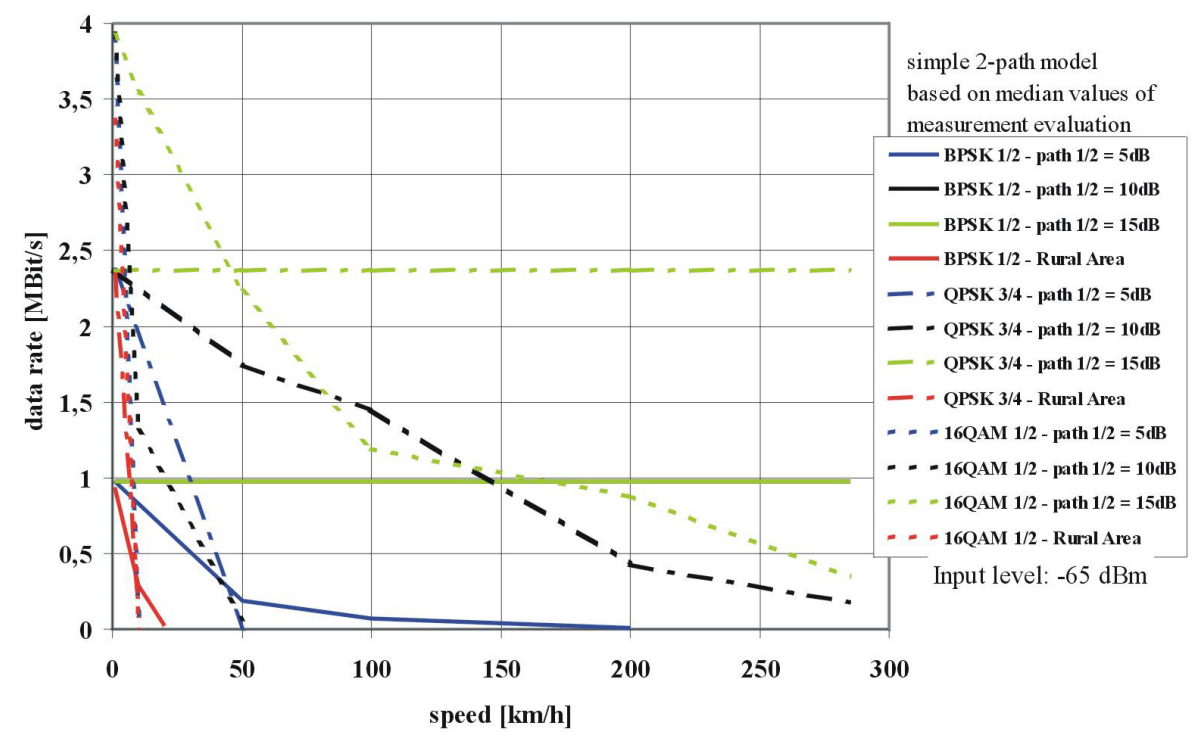

Fig. 9. Measured TCP/IP throughput as function of speed for a preliminary IEEE802.16 system operated at $3.5 \mathrm{GHz}$ with $3.5 \mathrm{MHz}$ bandwidth for different modulation schemes and different radio channels.

\section{References}

BWCS report: Railway Wireless LAN Services, BWCS limited, 2003.

http://www.medav.de

Martin, U.: Ausbreitung in Mobilfunkkanälen: Beiträge zum Entwurf von Messgeräten und zur Echoschätzung, Dissertation Universität Erlangen, 1994. 